\section{Elastase-Induced Rabbit Aneurysms Model Complicated by Thoracic Aortic Aneurysms}

Published reports have described the elastase-induced aneurysm model in rabbits as easy and reproducible and possessing geometric, pathologic, and hemodynamic features that are similar to those of human cerebral aneurysms. ${ }^{1}$ We used a simple surgical technique to produce the elastase-induced aneurysm model in rabbits ${ }^{2}$ and used it to explore the efficacy of new endovascular devices. Recently, we found that 3 rabbits unexpectedly developed a thoracic aortic aneurysms (TAA), which was located in the posterior part of thoracic aorta, in 40 elastase-induced rabbit aneurysm models (Fig 1).

In contrast to traditional techniques, we used a temporary arcuated aneurysm clip rather than an occlusion balloon to achieve flow arrest in the right common carotid artery (RCCA). When we checked the primary records of the elastase-induction procedure in the 3 cases, we found that the stump of the RCCA had collapsed in the first few minutes after complete filling with elastase had been achieved. We deduced that when the origin of the RCCA was separated from the surrounding tissue in the initial procedure, $\geq 1$ arterial branch originating from the proximal RCCA that was barely visible to the naked eye had split, allowing elastase to leak into the tissue surrounding the thoracic aorta during incubation, ultimately resulting in TAA formation. Thiex et $\mathrm{al}^{3}$ recommended avoiding elastase leaking into the branch originating from the proximal RCCA because of the danger of lethal hemorrhage of the trachea. ${ }^{3}$ We are unable to explain how the leaked elastase came into contact with the thoracic aorta and thereby induced TAA, because the TAAs we have reported here were in the pericardial cavity. It is possible that elastase permeated into the pericardial cavity and concentrated around the posterior part of thoracic aortic artery during the surgical procedure.

The TAAs we found here in rabbits reproduced key features of human TAAs in regard to site-specific phenotypes and pathology. The external surroundings of aneurysms in the pericardial cavity resembled those of the intracranial subarachnoid cavity, and the location is similar to that of human ophthalmic segment aneurysms.

This case report comes from an animal experimental study, which was supported by the China Postdoctoral Science Foundation (grant 20100481521). All procedures of the animal experimental study in this article were approved by the Institutional Animal Care and Use Committee at our hospital.

\section{References}

1. Zeng Z, Kallmes DF, Durka MJ, et al. Hemodynamics and anatomy of elastaseinduced rabbit aneurysm models: similarity to human cerebral aneurysms? AJNR Am J Neuroradiol 2011;32:595-601

2. Wang K, Huang Q, Hong B, et al. Neck injury is critical to elastase-induced aneurysm model. AJNR Am J Neuroradiol 2009;30:1685-87

3. Thiex R, Hans FJ, Krings T, et al. Haemorrhagic tracheal necrosis as a lethal
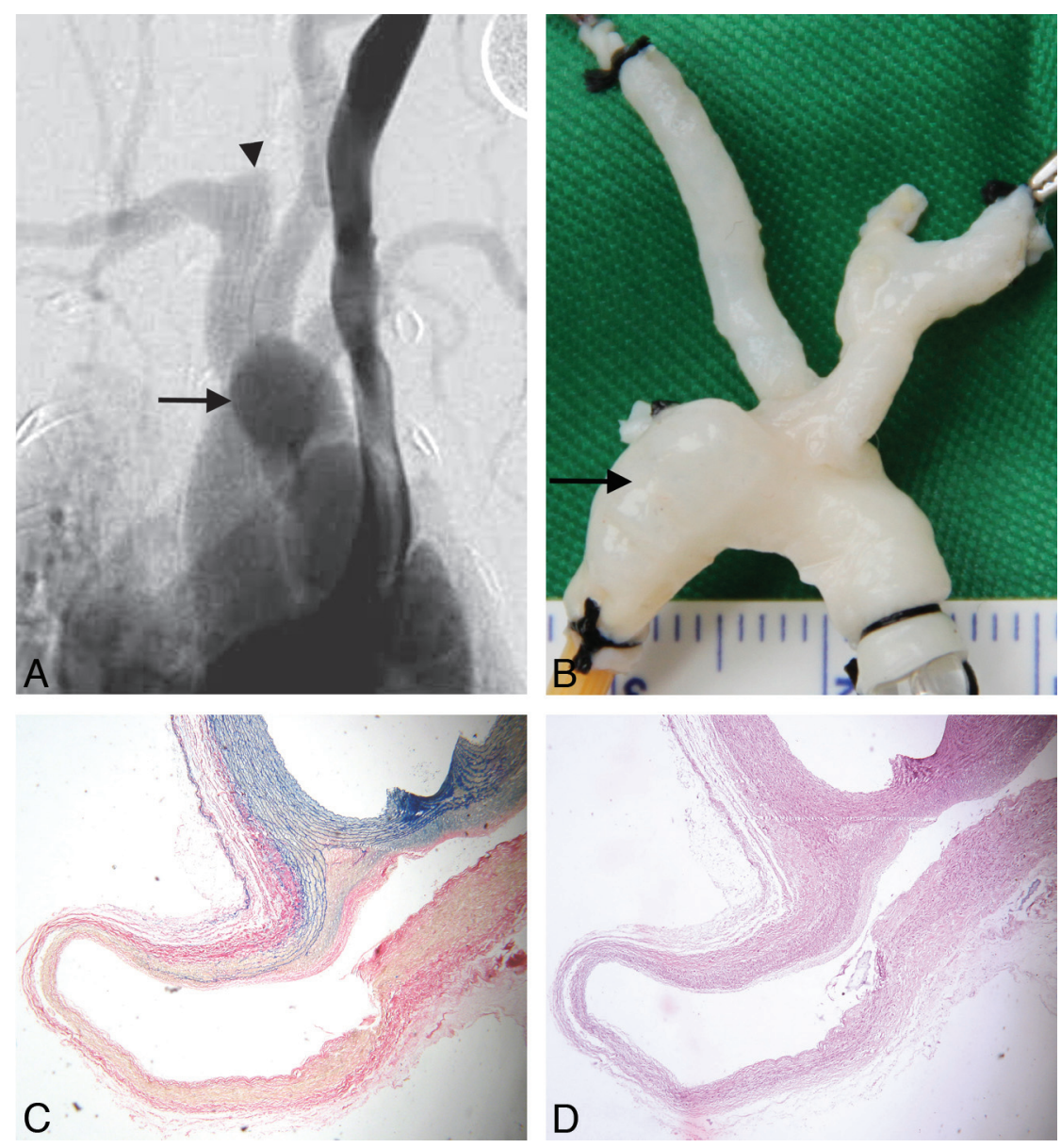

Fig 1. Elastase-induced rabbit aneurysm complicated by a TAA. $A$, Intravenous DSA shows an aneurysm (arrowhead) in the right subclavian artery and a TAA in the thoracic aorta (arrow). B, Macroscopically, the TAA (arrow) is located in the posterior part of the aortic arch and descending thoracic aorta (posteroanterior view). C, A specimen stained with Ponceau/Victoria Blue shows the normal elastic layers of the thoracic aorta as wavy blue lines. Abrupt termination of the elastic lamina at the dissection margins of the TAA is evident (original magnification, $\times 40$ ). D. Hematoxylin-eosin staining shows a thin aneurysm wall, the absence of an inflammatory reaction, and marked loss of cellular elements (original magnification, $\times 40$ ). 
complication of an aneurysm model in rabbits via endoluminal incubation with elastase. Acta Neurochir (Wien) 2004;146:285-89, discussion 289

K. Wang

S. Yuan

Department of Neurosurgery

General Hospital of Jinan Military Command Jinan, China

http://dx.doi.org/10.3174/ajnr.A3095 